\title{
Antibiotic prescribing after successful drainage of dentoalveolar infections
}

An outcome audit of the treatment of acute dentoalveolar infection: impact of penicillin resistance

T. Kuriyama, E. G. Absi, D. W. Williams and M. A. 0. Lewis Br Dent J 2005; 198: 759-763

\section{Objective}

The aim of this audit was to measure the outcome of treatment of acute dentoalveolar infection and to determine if this was influenced by choice of antibiotic therapy or the presence of penicillin-resistance.

\section{Subjects and methods}

A total of 112 patients with dentoalveolar infection were included in the audit. All patients underwent drainage, either incisional $(n=105)$ or opening of the pulp chamber $(n=7)$ supplemented with antibiotic therapy. A pus specimen was obtained from each patient for culture and susceptibility. Clinical signs and symptoms were recorded at the time of first presentation and re-evaluated after 48 or $72 \mathrm{~h}$.

\section{Results}

A total of 104 (99\%) of the patients who underwent incisional drainage exhibited improvement after $72 \mathrm{~h}$. Signs and symptoms also improved in five of the seven patients who underwent drainage by opening of the root canal although the degree of improvement was less than that achieved by incisional drainage. Penicillin-resistant bacteria were found in 42 (38\%) of the 112 patients in this study. Of the 65 patients who were given penicillin, 28 had penicillin-resistant bacteria. There was no statistical difference in the clinical outcome with regard to the antibiotic prescribed and the presence of penicillin-resistant bacteria. Strains of penicillin-resistant bacteria were isolated more frequently in patients who had previously received penicillin $(p<0.05)$.

\section{Conclusion}

Incisional drainage appeared to produce a more rapid improvement compared to drainage by opening of the root canal. The presence of penicillin-resistant bacteria did not adversely affect the outcome of treatment. The observations made support surgical drainage as the first principle of management and question the value of prescribing penicillin as part of treatment.

\section{IN BRIEF}

- Incisional drainage is the first principle in management of acute dentoalveolar infection.

- Penicillin-resistant bacteria are often present in acute dental infection.

- The presence of penicillin resistant bacteria does not adversely affect the outcome of treatment even if penicillin is prescribed.

- It is likely that antibiotic therapy is often prescribed unnecessarily in treatment of acute dental infection.

\section{COMMENT}

Systemic antibiotics may limit spread of infection and complications, but it is preferable to prescribe on the basis of a culture and sensitivity, which can take several days. Antibiotic prescribing remains common practice, despite universal recognition that achieving drainage is the goal. There has been an increase in penicillin-resistant organisms in odontogenic infections, thus it is questionable whether penicillin should be prescribed, unless there is a clear indication.

The study measured signs and symptoms (pain, swelling, temperature) on a 4-point scale, highlighting the importance of sterile aspiration, prior to incision and rapid transfer to the laboratory. The latter may not be available to a GDP. Equally, comprehensive microbiological investigations or expertise may not always be available outside a dental teaching hospital. Data were collected from 112 patients: 15\% had varying degrees of pyrexia and six different antibiotic regimes were reported. Improvement in the signs and symptoms was not influenced by the infected tooth site. The article provides a comprehensive description of the variety of cultured bacteria.

Almost $50 \%$ of patients had been administered antibiotics prior to incision and drainage. 53\%, who had received penicillin in the past, had penicillin-resistant bacteria in their cultures. This was significantly higher than in patients who had not previously received penicillin. Over $50 \%$ of the patients received penicillin after surgical drainage. Almost $50 \%$ of cultures contained penicillin-resistant organisms.

Important principles when dealing with dentoalveolar infections include achieving surgical drainage and addressing the need for adjunc-tive antibiotic therapy. It is accepted that it is preferable to achieve drainage through the soft tissues, gaining direct access to the abscess cavity. Drainage through the root canal may be compromised by repeated blockage and poor quality specimens. Important questions are raised about the role of antibiotics: why were they prescribed where drainage had been achieved, given that only $15 \%$ of the patients had varying degrees of pyrexia? It would be useful to compare patients treated with drainage alone with those where drainage and adjunctive antibiotic therapy is common practice. Anti-biotic prescribing is usual in cases where there is general malaise, significant pyrexia and gross swelling with trismus.

Where it is impossible to establish drainage, antibiotics may be required, but penicillin-resistant organisms may play a role. Investigation of the apparent increase in antibiotic resistance in bacterial infections is required. A higher incidence of penicillin-resistant Prevotella species was recorded in patients with a history of penicillin therapy. These organisms appear to afford protection for other organisms which are not penicillin-resistant. Clear conclusions are drawn from this study which purports to be the first recorded audit of treatments for dentoalveolar infection. The appropriateness of treatment with penicillin or any other antibiotic if drainage has been established is questioned. The authors highlight the importance of future studies to identify strict criteria for adjunctive antibiotic therapy in the treatment of dentoalveolar infection where adequate drainage has been achieved.

J. G. Cowpe, Head of Division of Oral \& Maxillofacial Surgery, Bristol Dental School

doi: 10.1038/sj.bdj.4812448 